\title{
Characterization of a pollen-specific agpl-like protein in Arabidopsis thaliana
}

\author{
Tian $\mathrm{Wu}^{*}$, Feng Feng, Changhui Ye and Yingzhi Li \\ Agricultural College, Guangdong Ocean University, Guangdong, China.
}

\begin{tabular}{|c|c|}
\hline ARTICLE INFO & ABSTRACT \\
\hline $\begin{array}{l}\text { Article history: } \\
\text { Received on: } 16 / 05 / 2016 \\
\text { Revised on: } 10 / 06 / 2016 \\
\text { Accepted on: } 20 / 06 / 2016 \\
\text { Available online: } 05 / 11 / 2016\end{array}$ & $\begin{array}{l}\text { In plants, pollen tube germination occurs widely in flowering plants. In Arabidopsis thaliana, it has been reported } \\
\text { ARABINOGALACTAN PROTEIN 1 (AGP1) plays an important role in pollen tube germination. The } \\
\text { expression of arabinogalactan protein in Arabidopsis pollen tubes has been extensively studied. Herein, we } \\
\text { characterized an Arabidopsis AGP1-LIKE PROTEIN (ALP), which is shown to have } 47 \% \text { homology at the } \\
\text { amino acid level to At1g24520.1 (BCP1), POLLEN PROTEIN1 in Brassica campestris. BCP1, which is highly }\end{array}$ \\
\hline $\begin{array}{l}\text { Key words: } \\
\text { agp1-like protein, } \\
\text { Arabidopsis, development, } \\
\text { pollen, pollen tube, siliques. }\end{array}$ & $\begin{array}{l}\text { expressed in both tapetum and microspores, is essential for pollen fertility. Transgenic Arabidopsis transtormed } \\
\text { with an } A L P \text { promoter-driven } \beta \text {-glucuronidase (GUS) construct exhibited strong GUS activity in the pollen and } \\
\text { young siliques, in good agreement with the RT-qPCR analysis. To further understand the function of ALP, the } \\
\text { ALP-RNAi lines were generated for further investigations. Phenotypic deficiency of siliques was observed in the } \\
\text { ALP-RNAi line, in comparison to the wild type. Furthermore, scanning electron microscopy suggested defects in } \\
\text { the ALP-RNAi line and in vivo pollen germination showed reduced ability in the ALP-RNAi line. Taken } \\
\text { together, our results suggested the important role of ALP in pollen and seed development. }\end{array}$ \\
\hline
\end{tabular}

\section{INTRODUCTION}

In flowering plants, pollen grains and embryo sacs represent the extremely reduced haploid generation, exerting sexual reproduction [1]. Successful fertilization event occurs when the pollen grain extrudes through a pore and grows through the pistil until it finds the female embryo sac in the ovule [2-5]. The pollen tube subsequently bursts and releases the sperm cells and fertilizes with a different nucleus in the female gametophyte [6-8]. This is a complex process, which includes a network with a variety of signaling events $[6,9,10]$. In Arabidopsis, it has been reported that arabinogalactan proteins (AGPs), were detected in stigma exudates, style transmitting tissues and pollen $[11,12]$. They serve as recognition signals, nutritional supply or guidance for the pollen tube $[13,14]$. AGPs have been identified as a group of hydroxyproline-rich glycoproteins, differentially expressed throughout plant development [12-15]. Previous studies have shown that AGPs were identified in pollen tubes of several plant species, and tube growth was affected by the Yariv phenyl

\footnotetext{
* Corresponding Author

Tian Wu, Agricultural College, Guangdong Ocean University, Guangdong, China. Email: wutian702@126.com
}

glycoside (a reagent which binds specifically to AGPs) [16]. These experimental evidence suggests that AGPs play an important role in pollen tube extension or guidance [17]. Analysis of the Arabidopsis thaliana genome revealed that 47 genes code for AGP polypeptide backbones [15]. "Classical" AGPs include an $N$ terminal secretion sequence, a central domain rich in Pro/Hyp and a $C$-terminal hydrophobic domain for signaling [12,14,15,17] Herein, we characterized an Arabidopsis AGP1-LIKE PROTEIN (ALP; At3g26110), which have been shown to have $47 \%$ homology at the amino acid level to At1g24520.1 (BCP1), POLLEN PROTEIN1 in Brassica campestris [18]. Previous reports have shown that $\mathrm{BCP} 1$ was highly expressed in tapetum and microspores and it is essential for pollen fertility $[18,19]$. Herein, transgenic Arabidopsis transformed with an ALP promoterdriven $\beta$-glucuronidase (GUS) construct and RT-qPCR analyses were utilized to study the expression profile of $A L P$.

To further understand the function of ALP, ALP-RNAi transgenic lines were generated before further used for observation. Phenotypic deficiency of siliques was detected in the ALP-RNAi line, in comparison to the wild type. Furthermore, scanning electron microscopy suggested defects in the ALP-RNAi line and in vivo pollen germination showed reduced ability in the ALPRNAi line. 


\section{MATERIALS AND METHODS}

\subsection{Plant Material and Growth}

Seeds (A. thaliana ecotype Columbia-0) were sterilized by treatment with $70 \%$ ethanol for $2 \mathrm{~min}$, $5 \%$ sodium hypochlorite for $5 \mathrm{~min}$, and several washes with $\mathrm{ddH}_{2} \mathrm{O}$. Subsequently, they were sowed on Murashige and Skoog basal medium agar plates and stratified in darkness at $4^{\circ} \mathrm{C}$ for 2 days. The seeds were germinated under continuous light for 2 weeks and subsequently potted in soil at $23^{\circ} \mathrm{C} / 21^{\circ} \mathrm{C}(16 \mathrm{~h}$ day/ $8 \mathrm{~h}$ night $)$ cycles.

\subsection{RT-qPCR analysis}

Samples containing $50 \mathrm{mg}$ of seedlings, rosette leaves (4week-old), stems, roots, open flowers and siliques was collected for RNA isolation using the RNeasy Isolation Kit (Qiagen). Extracted RNA were treated with DNase and reverse-transcribed to cDNA according to the procedure of the cDNA Synthesis Kit (Takara).

The expression of $A L P$ genes was detected by RT-qPCR using the following primer pairs: ALP-qRT-F (ATGGCGCGTCTTCACCTAGCTCTCCT) and ALP-qRT-R (TCAGTTGTTCCAATAGCATTGTCATCAGC). StepOne Plus (Applied Biosystems) and FastStart Universal SYBR Green Master (Roche) were utilized for RT-qPCR. Conditions for RTqPCR were as follows: $95^{\circ} \mathrm{C}, 10 \mathrm{~min} ; 40$ cycles of $95^{\circ} \mathrm{C}, 15 \mathrm{~s}$ followed by $60^{\circ} \mathrm{C}, 1 \mathrm{~min}$. For each sample, three replicates were performed.

\subsection{Generation of $A L P$ pro::GUS and ALP-RNAi lines in Arabidopsis}

The 5'-flanking region of ALP (At3g26110) was amplified by PCR with following primers: ALPpro-F (GCTGGATCCAAACCCTAAGCTACATTTTGTGG) and ALPpro-R (GCTCCCGGGTAATACCAGAGTTGATATTTTCC GACG). The fragment was purified and cloned into the pGEM-T Easy Vector system (Promega) to yield plasmid and was subcloned into corresponding restriction sites on the binary vector pBI101.3 (Clontech) to generate ALPpro::GUS fusion plasmids. For the RNAi construct, a DNA fragment of the $A L P$ cDNA was amplified by PCR using primes ALP-RNAi-F (GCTGAAATCCTTCAAGATAAATAAATAAAACAAAATAC CCATAAAACATT) and ALP-RNAi-R (GCTGATATCTAAAAAACTTTCTTTATATTAGTATATTAA ATATGTATCATCC), clonedinto the pGEM-T Easy Vector cut with $X m n \mathrm{I}$ and $E c o R \mathrm{~V}$, and transferred into the vector pHELLSGATE8.

Subsequently, the recombinant plasmids were used in Agrobacterium tumefaciens transformation of wild-type Arabidopsis Col-0 by the floral dip method [20]. Seeds from $\mathrm{T}_{0}$ plants were first screened using MS plants containing kanamycin antibiotics. The transformants barboring kanamycin-resistance were subsequently identified by PCR analysis. $\mathrm{T}_{3}$ stable transgenic plants expressing ALPpro::GUS fusion were used for GUS assays.

\subsection{GUS assays}

GUS histochemical assays were performed to detect the expression of $A L P[21]$. Briefly, samples of inflorescences, mature flowers, pollens, 6-week-old siliques, 8-week-old stems, 3-weekold rosette and 9-day-old seedlings from transgenic Arabidopsis expressing the ALPpro::GUS constructs were stained with GUS staining solution (100 mM sodium phosphate buffer, $\mathrm{pH} 7.0,0.1 \%$ Triton $\mathrm{X}-100,2 \mathrm{mM}$ potassium ferricyanide, $2 \mathrm{mM}$ potassium ferrocyanide, $1 \mathrm{mg} \mathrm{mL}$ 5-bromo-4-chloro-3-indolyl- $\beta$-Dglucuronide).

Transgenic Arabidopsis samples and the pBI101.3transformed control were infiltrated in GUS staining buffer by vacuuming for $1 \mathrm{~h}$, followed by a $4 \mathrm{~h}$ incubation. Chlorophyll was removed by washing in $70 \%$ ethanol, and the samples were directly photographed by Nikon 80i Microscope.GUS activity on the quantification of 4-methylumbelliferone (MUG)was normalized to protein concentration as nmole of product generated per mg of total protein per minute. In each experiment, at least five leaves were stained following treatment.

\subsection{Scanning electron microscopy}

Five-week-old Arabidopsis Col-0 and ALP-RNAi pollen were utilized for observation. Pollen were air-dried for 3 days, and were mounted onto standard aluminium stubs and sputter coated with gold particles using six 30-s bursts, and observed with a scanning electron microscope (Hitatchi S3400).

\subsection{In vivo pollen tube germination}

For the in vivo pollen tube germination [22], the preemasculated mature Col-0 (wild-type) pistils were pollinated with pollen from Col-0 or transgenic ALP-RNAi line. The pollinated pistils were collected $16 \mathrm{~h}$ after pollination and immediately fixed in a solution of 1:3 acetic acid to ethanol for $2 \mathrm{~h}$ and cleared in $1 \mathrm{M} \mathrm{NaOH}$ overnight $(16 \mathrm{~h})$. Pistils were placed into $1 \%$ aniline blue solution and allowed to stain for $5 \mathrm{~h}$ in dark before being mounted on slides. Observation and imaging were taken using a Nikon 80i Microscope (emission spectra: 440 612 $\mathrm{nm})$

\section{RESULTS AND DISCUSSION}

\subsection{ALP was expressed in the pollen}

The microarray database e-FP brower revealed that $A L P$ was not highly expressed in any tissues other than those of the flower mature pollen. The expression of $A L P$ was subsequently investigated by RT-qPCR (Fig. 1A). The results from the RTqPCR revealed that highest expression of $A L P$ occurred in the flower buds and open flowers (Fig. 1A). Expression of $A L P$ in root, rosette leaves and stems was significantly reduced (Fig. 1A), which is also consistent with the microarray results from Genevestigator (https://genevestigator.com/gv/; Fig. 1B). To investigate the spatial and temporal expression pattern of $A L P$, a $1.2-\mathrm{kb} 5$ '-flanking fragment of $A L P$ was PCR-amplified and cloned into the $\mathrm{pBI} 101.3$ binary vector. 
A

B
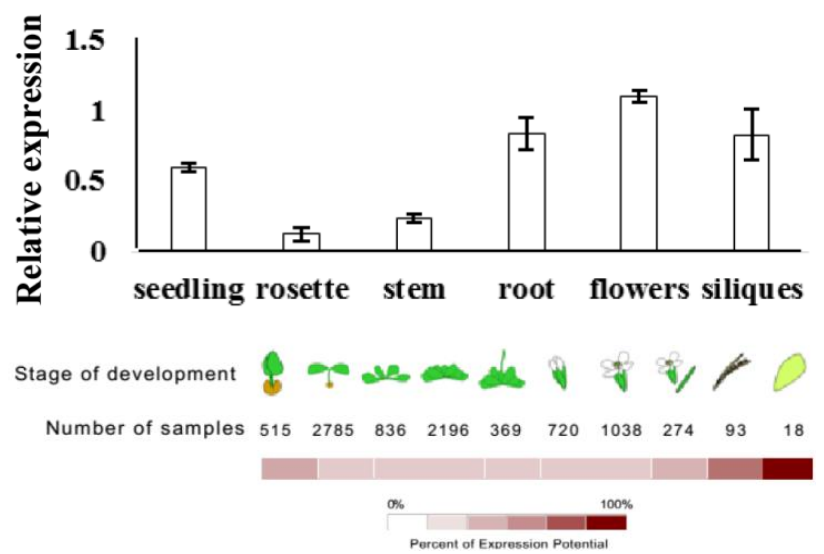

Fig. 1: Expression of ALP in Arabidopsisthaliana.

(A) Quantitative real-time PCR analysis of $A L P$ expression in seedlings, rosette leaves (4-week-old), stems, roots, open flowers and siliques. Results were

normalized against the expression of ACTIN. Each bar represents a mean value of five repeats from two independent biological samples \pm SE.

(B) The expression of $A L P$ in different developmental stages were carried out by analysis of the microarray data by Genevestigator

(https://genevestigator.com/gv/).

Histochemical staining showed that GUS driven by the $A L P$ promoter was highly expressed in the pollen (Fig. 2). Interestingly, GUS expression was also observed in the pollen tube as observed by in vitro pollen tube germination (Fig. 2D).
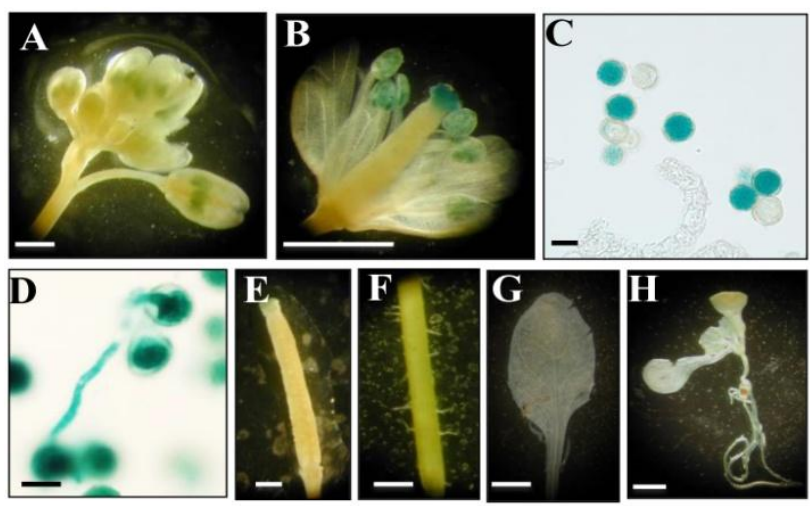

I

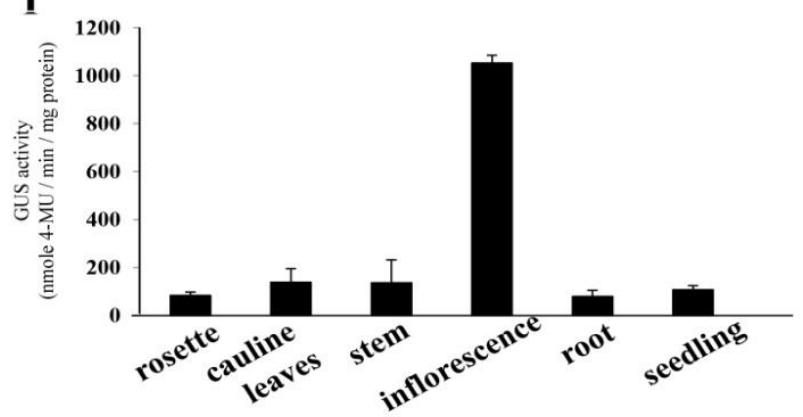

Fig. 2: Expression of $A L P$ in Arabidopsis.

Histochemical staining shows GUS expression of ALPpro::GUS including inflorescences (A), mature flowers (B), pollens (C) and germinating pollen tube (D). Different GUS staining of other tissues are also shown, including 6-weekold siliques (E), 8-week-old stems (F), 3-week-old rosette (G) and 9-day-old seedlings $(\mathrm{H}) . \mathrm{Bar}=2 \mathrm{~mm}$ in $(\mathrm{A}),(\mathrm{B}),(\mathrm{E}),(\mathrm{F}),(\mathrm{G})$ and $(\mathrm{H}), 20 \mu \mathrm{m}$ in $(\mathrm{C})$ and (D). (I) Quantitative fluorimetric measurement of GUS activity in various tissues (4-week-old rosette and cauline leaves, 6-week-old stem and inflorescences, 3-week-old root and seedlings) from ALPpro::GUS. Average values were obtained from experiments performed with 3-5 independent lines per construct, each line represented by $8-10$ individual plants. Bars indicate the standard errors of three replicates.

\subsection{ALP affects pollen and seed development}

To investigate the function of ALP in seed development, loss-of-function transgenic plants were needed. However, the location of the T-DNA insertion in two alp mutant lines (SALK_006192 and SALK_006065) is at the 3'-UTR. Given high possibility of null efficiency in these two mutant, ALP-RNAi transgenic Arabidopsis lines was generated and characterized followed by the observation of the pollen and silique morphology. When the siliques from Col-0 and ALP-RNAi were examined, some 'shorter' siliques (arrowheads in Fig. 3A) were observed in the ALP-RNAi plants. When seed number per silique was counted, significant differences between the wild type and ALP-RNAi were detected (Fig. 3B), suggesting that fertility had been altered in ALP-RNAi.
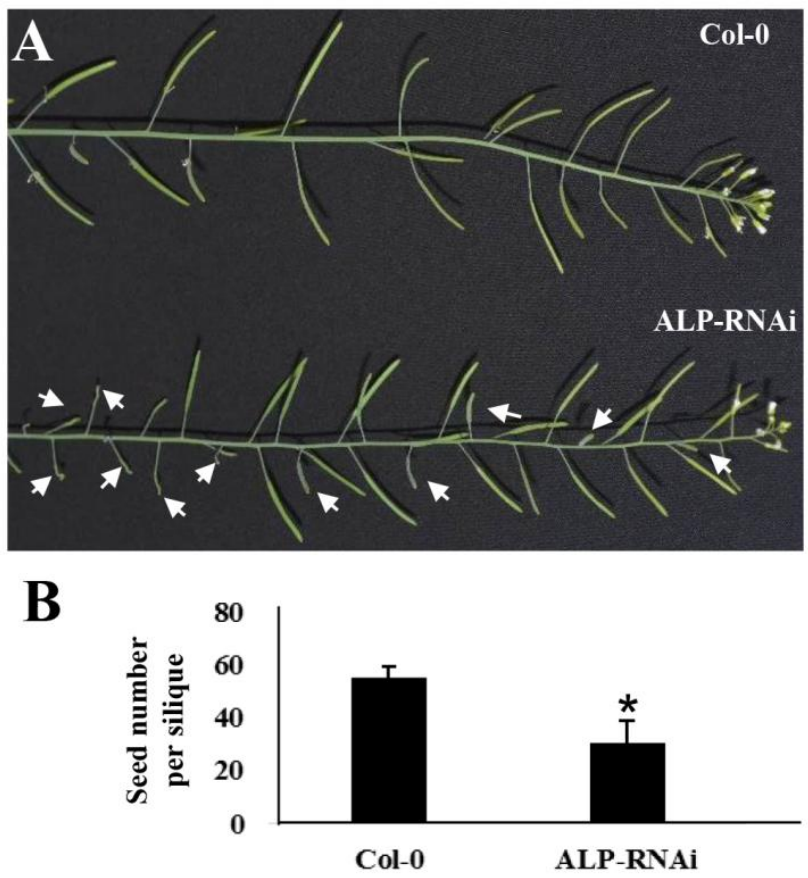

Fig. 3: Comparision of the silique phenotypes between Col-0 and ALP-RNAi plants.

(A) The inflorescences from 6-week-old Col-0 and ALP-RNAi Arabidopsis genotypes were compared. Some siliques from ALP-RNAi were shorter (white arrows) compared with those of Col-0. (B) Statistical analysis of seed number per silique. Siliques of Col-0 and ALP-RNAi were taken and their seeds counted. Values are means $\pm \mathrm{SD}, \mathrm{n}=20$; asterisk denotes significant differences from the wild type $(P<0.0 .1 ; \mathrm{n}=30)$ by the Student $\mathrm{t}$-test.

Furthermore, scanning electron microscopy (SEM) revealed that a $40 \%$ of defective pollen grains of ALP-RNAi in comparison with the wild type (Fig. 4A). Besides morphological analysis, the pollen activity of the ALP-RNAi pollen was compared with that of the wild type to examine for physiological changes. In vivo pollen germination assays were carried and 
observed. After $16 \mathrm{~h}$ incubation, the pollen tubes of the "wild-type pistil pollinated with ALP-RNAi pollen" were noticeably shorter (Fig. 4B) than those of the "wild-type pistil pollinated with ALPRNAi pollen" (Fig. 4B), suggesting that physiological changes in the ALP-RNAi pollen had accompanied its morphological changes.
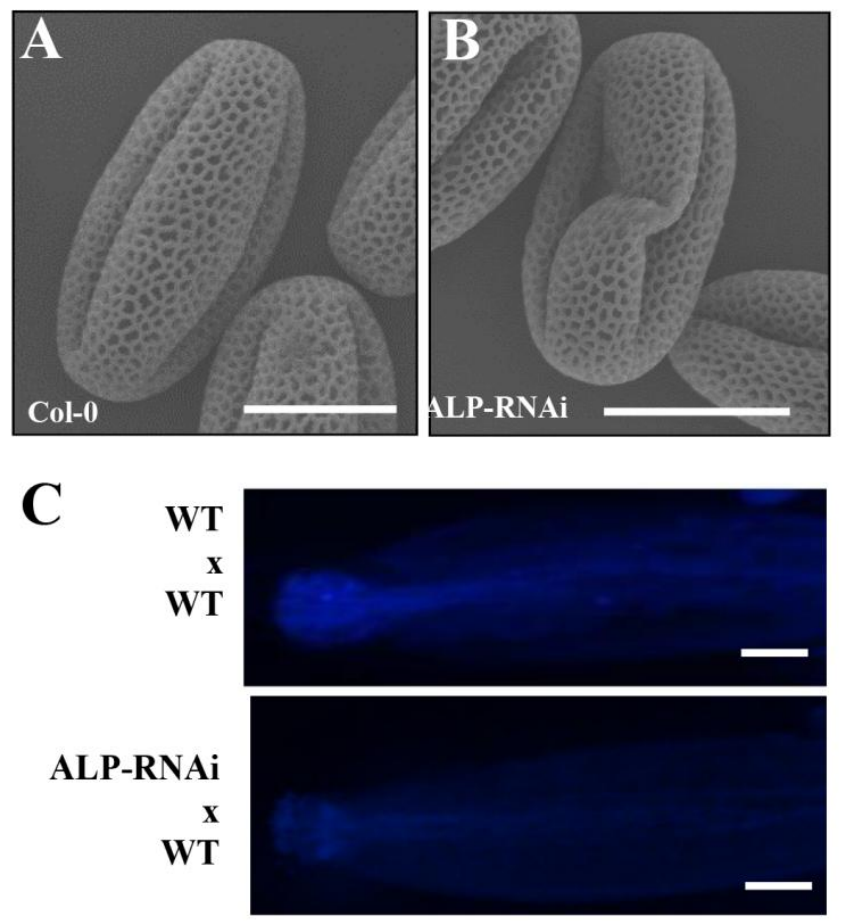

Fig. 4: Comparision of the pollen phenotypes between Col-0 and ALP-RNAi plants. Scanning electron microscopy were performed to analyze pollen from the Arabidopsis Col-0 (A) and ALP-RNAi (B). (C) The ALP-RNAi is defective in pollen tube germination. The wild-type $(\mathrm{Col}-0)$ pistils were pollinated with the Col-0 or ALP-RNAi pollen and incubated for $16 \mathrm{~h}$ before aniline blue stain. $\operatorname{Bar}=10 \mu \mathrm{m}$ in $(\mathrm{A})$ and $(\mathrm{B}) ; 100 \mu \mathrm{m}$ in $(\mathrm{C})$.

\subsection{Possible role of ALP in pollen development}

ALP is shown to have $47 \%$ homology at the amino acid level to At1g24520.1 (BCP1), POLLEN PROTEIN1 in Brassica campestris. BCP1 was found to have a diploid/haploid mode of action which control male fertility at both sporophytic and gametophytic levels based on the observation that expression perturbation of BCP1 in the tapetum and pollen caused pollen development arrest at the uninucleate microspore stage and later at the bicellular pollen stage respectively [18]. The production of viable pollen grains is vital for male fertility in flowering plants due to their ability to produce and deliver male gametes and sperm cells to embryo sacs [23]. Gene specifically expressed in the anthers likely control male fertility [18]. ALP, an anther-specific protein, which is highly expressed in pollen and siliques, may have functions regarding male fertility. Besides, ALP protein was predicted to localize at the ER with high scores (0.99) using Predotar v.1.03 (https://urgi.versailles.inra.fr/predotar/ predotar.html). The putative transmembrane domains and functional domain of ALP were predicted using TMHMM server v. 2.0 (http://www.cbs.dtu.dk/services/TMHMM/) and SMART (http://smart.embl-heidelberg.de/). Both programs reveal a single peptide (a.a. 1-22) and a transmembrane domain (a.a. 108-127) in ALP (Supplementary Fig. S1). The ALP $N$-terminal signaling peptide was also predicted by iPSORT (http://ipsort.hgc.jp/) and TargetP1.1 (http://www.cbs.dtu.dk/services/TargetP/). These predictions suggest that ALP may undergo the secretory pathway.

While a growing number of genes have been identified controlling male fertility [1,6,8,23-28], still, relatively little is known about the genetic mechanism that governs male fertility. Most of the studies focus on pollen development and structures [23,29-31]. Arabidopsis LESS ADHESIVE POLLEN5 (LAP5) and $L A P 6$ encode anther-specific proteins, which were reported to be expressed in young anthers and in flower buds (stages 9 and 10) and they are essential for pollen exine development [32]. Mutation of either gene led to abnormal exine patterning, while the double mutant showed sterility due to a lack of exine deposition and subsequently collapsed pollen [32]. To sum up, our studies provide clues for the pollen-expression of ALP in Arabidopsis, which hints a new avenue for further investigations of its role in pollen development.

\section{CONCLUSION}

Based upon our findings, $A L P$ was expressed in the pollen of Arabidopsis. Phenotypic deficiency of siliques was observed in the ALP-RNAi line, in comparison to the wild type. Furthermore, scanning electron microscopy suggested defects in the ALP-RNAi line and in vivo pollen germination showed reduced ability in the ALP-RNAi line. Taken together, our results revealed the $A L P$ expression in Arabidopsis pollen, and the deficiency observed in ALP-RNAisuggested the important role of ALP inpollen and seed development.Hence, it is worthwhile for further investigation on the detailed mechanisms of ALPinvolvement in pollen and seed development,

\section{ACKNOWLEDGEMENTS}

Financial support and sponsorship: This work was partially supported by Science and Technology Planning Project of Guangdong Province, China (2013B020304003 and 2014A020208137).

Conflict of Interests: There are no conflicts of interest.

\section{REFERENCES}

1. Borg M, Brownfield L, Twell D. Male gametophyte development: a molecular perspective. J Exp Bot.2009; 60: 1465-1478.

2. Piffanelli P, Ross JH, Murphy D. Biogenesis and function of the lipidic structures of pollen grains. Sex Plant Reprod.1998; 11: 65-80.

3. Piffanelli P, Ross JH, Murphy DJ. Intra- and extracellular lipid composition and associated gene expression patterns during pollen development in Brassica napus. Plant J. 1997; 11: 549-562.

4. Pina C, Pinto F, Feijo JA, Becker JD. Gene family analysis of the Arabidopsis pollen transcriptome reveals biological implications for cell growth, division control, and gene expression regulation. Plant Physiol. 2005; 138: 744-756. 
5. Quilichini TD, Friedmann MC, Samuels AL, Douglas CJ. ATPbinding cassette transporter G26 is required for male fertility and pollen exine formation in Arabidopsis. Plant Physiol. 2010; 154: 678690

6. Franklin-Tong VE. Signaling in pollination. Curr Opin Plant Biol. 1999; 2: 490-495.

7. Gu F, Nielsen E. Targeting and regulation of cell wall synthesis during tip growth in plants. J Integr Plant Biol. 2013; 55: 835-846.

8. Guan Y, Guo J, Li H, Yang Z. Signaling in pollen tube growth: crosstalk, feedback, and missing links. Mol Plant. 2013; 6: 10531064.

9. Farmer EE, Weber H, Vollenweider S. Fatty acid signaling in Arabidopsis. Planta. 1998; 206: 167-174.

10. Wang X. Lipid signaling. Curr Opin Plant Biol. 2004; 7: 329-336.

11. Aarts MG, Keijzer CJ, Stiekema WJ, Pereira A. Molecular characterization of the CERl gene of arabidopsis involved in epicuticular wax biosynthesis and pollen fertility. PlantCell. 1995; 7 : 2115-2127.

12. Coimbra S, Almeida J, Junqueira V, Costa ML, Pereira LG.Arabinogalactan proteins as molecular markers in Arabidopsis thaliana sexual reproduction. J Exp Bot. 2007; 58: 4027-4035.

13. Coimbra S, Jones B, Pereira LG. Arabinogalactan proteins (AGPs) related to pollen tube guidance into the embryo sac in Arabidopsis. Plant Signal Behav. 2008; 3: 455-456.

14. Seifert GJ, Roberts K. The biology of arabinogalactan proteins. Annu Rev Plant Biol. 2007; 58: 137-161.

15. Pereira AM, Masiero S, Nobre MS, Costa ML, Solis MT, Testillano PS, et al. Differential expression patterns of arabinogalactan proteins in Arabidopsis thaliana reproductive tissues. J Exp Bot.2017; 65: 5459-5471.

16. Willats W, Knox J. A role for arabinogalactan-proteins in plant cell expansion: evidence from studies on the interaction of $\beta$-glucosyl Yariv reagent with seedlings of Arabidopsis thaliana. Plant J.1996; 9: 919-925.

17. Majewska-Sawka A, Nothnagel E. The multiple roles of arabinogalactan proteins in plant development. Plant Physiol.2000; 122: 3-10.

18. Xu H, Knox RB, Taylor PE, Singh MB. Bcpl, a gene required for male fertility in Arabidopsis. Proc Natl Acad Sci USA 1995; 92: 2106-2110.

19. Theerakulpisut $\mathrm{P}, \mathrm{Xu} H$, Singh $\mathrm{MB}$, Pettitt JM, Knox RB. Isolation and developmental expression of Bcp1, an anther-specific cDNA clone in Brassica campestris. Plant Cell. 1991; 3: 1073-1084.

20. Clough SJ, Bent AF. 1998. Floral dip: a simplified method for Agrobacterium-mediated transformation of Arabidopsis thaliana. Plant J. 1998;16: 735-743.

21. Jefferson RA, Kavanagh TA, Bevan MW. GUS fusions: betaglucuronidase as a sensitive and versatile gene fusion marker in higher plants. EMBO J. 1987; 6: 3901-3907.

22. Taylor LP, Hepler PK. Pollen germination and tube growth. Annu.Rev.Plant Biol.1997; 48: 461-491.
23. Wilson ZA, Zhang DB. From Arabidopsis to rice: pathways in pollen development. J Exp Bot. 2009; 60: 1479-1492.

24. Ariizumi T, Toriyama K. Genetic regulation of sporopollenin synthesis and pollen exine development. Annu Rev Plant Biol. 2001; 62: $437-460$

25. Becker JD, Boavida LC, Carneiro J, Haury M, Feijo JA. Transcriptional profiling of Arabidopsis tissues reveals the unique characteristics of the pollen transcriptome. Plant Physiol. 2003; 133 713-725.

26. da Costa-Nunes JA, Grossniklaus U. Unveiling the gene-expression profile of pollen. Genome Biol. 2003; 5: 205.

27. Hirano K, Aya K, Hobo T, Sakakibara H, Kojima M, Shim RA, et al. Comprehensive transcriptome analysis of phytohormone biosynthesis and signaling genes in microspore/pollen and tapetum of rice. Plant Cell Physiol. 2008; 49: 1429-1450.

28. Mascarenhas JP. The Biochemistry of Angiosperm Pollen Development. Botanical Review. 1975; 41: 259-314.

29. Evans DE, Taylor PE, Singh MB, Knox RB. The interrelationship between the accumulation of lipids, protein and the level of acyl carrier protein during the development of Brassica napus L. pollen. Planta. 1992; 186: 343-354.

30. Johnson-Brousseau SA, McCormick S. A compendium of methods useful for characterizing Arabidopsis pollen mutants and gametophytically-expressed genes. Plant J. 2004; 39: 761-775.

31. McCormick S, Twell D, Vancanneyt G, Yamaguchi J. Molecular analysis of gene regulation and function during male gametophyte development. Symp Soc Exp Biol. 1991; 45: 229-244.

32. Dobritsa AA, Lei Z, Nishikawa S, Urbanczyk-Wochniak E, Huhman DV, Preuss D et al. LAP5 and LAP6 encode anther-specific proteins with similarity to chalcone synthase essential for pollen exine development in Arabidopsis. Plant Physiol. 2010; 153: 937-955.

\section{How to cite this article:}

Wu T, Feng F, Ye C, Li Y. Characterization of a pollen-specific agp1-like protein in Arabidopsis thalian. J App Biol Biotech. 2016; 4 (06): 010-014. DOI: 10.7324/JABB.2016.40602 